\title{
The effect of a short-wavelength mode on the evolution of a long-wavelength perturbation driven by a strong blast wave
}

\author{
A. R. Miles ${ }^{\text {a) }}$ \\ Lawrence Livermore National Laboratory, Livermore, California 94550 and University of Maryland, \\ College Park, Maryland 20741
}

M. J. Edwards, B. Blue, J. F. Hansen, and H. F. Robey

Lawrence Livermore National Laboratory, Livermore, California 94550

R. P. Drake, C. Kuranz, and D. R. Leibrandt

University of Michigan, 2455 Hayward Street, Ann Arbor, Michigan 48109

(Received 6 May 2004; accepted 24 June 2004; published online 4 November 2004)

\begin{abstract}
Shock-accelerated material interfaces are potentially unstable to both the Richtmyer-Meshkov and Rayleigh-Taylor (RT) instabilities. Shear that develops along with these instabilities in turn drives the Kelvin-Helmholtz instability. When driven by strong shocks, the evolution and interaction of these instabilities is further complicated by compressibility effects. This paper details a computational study of the formation of jets at strongly driven hydrodynamically unstable interfaces, and the interaction of these jets with one another and with developing spikes and bubbles. This provides a nonlinear spike-spike and spike-bubble interaction mechanism that can have a significant impact on the large-scale characteristics of the mixing layer. These interactions result in sensitivity to the initial perturbation spectrum, including the relative phases of the various modes, that persists long into the nonlinear phase of instability evolution. Implications for instability growth rates, the bubble merger process, and the degree of mix in the layer are described. Results from relevant deceleration RT experiments, performed on OMEGA [J. M. Soures et al., Phys. Plasmas 5, 2108 (1996)], are shown to demonstrate some of these effects. (C) 2004 American Institute of Physics. [DOI: 10.1063/1.1812758]
\end{abstract}

\section{INTRODUCTION}

In core-collapse supernovae, the sudden release of an enormous amount of energy near the star's center drives a strong blast wave out through layers of progressively less dense material. ${ }^{1,2}$ The transmission of a blast wave through an interface from a denser to a less dense material constitutes an unstable system. ${ }^{3}$ Preexisting perturbations on the interface grow to larger amplitude after passage of the shock due to two different mechanisms. First, the transmission of a shock through a perturbed interface results in vorticity deposition regardless of whether the traversal is from heavy to light or from light to heavy. The subsequent evolution of the vorticity field leads to perturbation growth, an effect called the Richtmyer-Meshkov (RM) instability. ${ }^{4,5}$ With the passage of a blast wave, the interface begins to decelerate after shock refraction. Since the pressure decreases monotonically with distance behind the shock front, there is an attendant reversal of pressure and density gradients $(\nabla P \cdot \nabla \rho<0)$ in the heavy to light case. As long as this condition is satisfied at the interface, it is unstable to the Rayleigh-Taylor (RT) instability. ${ }^{6,7}$ Under the influence of the RM and RT instabilities, interface perturbations grow into spikes of heavier material "falling into" lighter fluid and bubbles of lighter fluid "rising into" heavier fluid. Shear that develops along the growing spikes drives Kelvin-Helmholtz (KH) growth, which contributes both to the development of characteristic

${ }^{\mathrm{a}}$ Electronic mail: miles15@1lnl.gov mushroom caps at the spike tips and to the eventual breakup of the interface into a turbulent flow. In addition to corecollapse supernovae, these instabilities will be present during the implosion of inertial confinement fusion (ICF) ignition targets currently being designed for the National Ignition Facility (NIF). For double-shell targets, the resulting mixing of hot fuel with cooler shell material can in turn result in significant reduction or even complete elimination of thermonuclear yield. ${ }^{8}$ Laboratory astrophysics with lasers, which is motivated by both the diagnostic limitations inherent in observational astrophysics and the need to validate the codes used to model astrophysical systems, provides a link between the two classes of applications.

The question of the dependence of RM and RT growth on the initial modal spectrum is at the heart of both astrophysical and ICF applications of compressible mix. This is particularly true for the deep nonlinear and transitional regimes, where linear and weakly nonlinear theories have long since become inapplicable but the similarity-based scaling arguments commonly applied to the turbulent regime are not yet necessarily valid. The deep nonlinear and transitional regimes must therefore bridge the gap between the earlier phases, where initial conditions (IC) have a strong and direct influence on the perturbation growth, and the turbulent regime perhaps characterized by self-similar growth independent of the initial spectrum. Most astrophysical systems of interest are not only turbulent but are very highly turbulent from very early times. For example, the Reynolds number in core-collapse supernovae is estimated ${ }^{9}$ to be of order $10^{10}$, 
many orders of magnitude above the value of $\sim 10^{4}$ suggested by Dimotakis ${ }^{10}$ as sufficient for the mixing transition. According to the analysis by Robey et al. of transition in nonstationary flows, transition in supernovae should occur in about $2.8 \mathrm{~s}$ after the instability initiation. ${ }^{11}$ In simulations by Fryxell, Muller, and Arnett of SN 1987A, perturbations on the $\mathrm{He} / \mathrm{H}$ interface did not grow to significant amplitudes until times of order $10^{3} \mathrm{~s}$ after the explosion while times of interest extend another order of magnitude higher. ${ }^{12}$ If an initial-condition-independent self-similar regime is ever to be attained at a blast-wave-driven interface, it must occur virtually instantaneously in a core-collapse supernova if the initial condition are amenable. However, the same cannot be said of the laser-driven high energy density experiments designed to study such astrophysical events. Present-day experiments can be very nonlinear but are often not turbulent, with Reynolds number much lower than in their astrophysical counterparts (of order $10^{5}$ for those designed to study aspects of supernova hydrodynamics ${ }^{11}$ ) and time scales that are not long compared to predicted transition times. Even if the one-dimensional hydrodynamics is appropriately scaled, the real relevance of "supernova-relevant" experiments is limited by whether or not the level of their dependence on initial conditions is similar to the dependence present in actual supernovae. If the complex nonlinear hydrodynamic mixing in supernovae is in fact initial-condition independent while current experiments are not, then future experiments should move to higher Reynolds numbers and longer time scales.

Our intent is to study the dependence on initial conditions of blast-wave-driven unstable interface evolution in two phases. In this paper, we consider mostly two-mode interfaces in the regime of recent and current experiments on OMEGA, ${ }^{13}$ and for such cases present a mechanism whereby the unstable evolution of a strongly driven perturbed interface can depend critically on details of the initial mode spectrum. Specifically, we consider how the evolution of a longwavelength mode is affected by a single short-wavelength component and the dependence of this effect on the relative phases of the two modes. In the second phase, and in a later paper, we will present a study of the dependence on initial conditions for many-mode interfaces under NIF-like drive conditions, in which the degree of phase coherence as well as the spectrum is varied.

We begin with brief descriptions of the experimental setup, relevant previous experiments, the $2 \mathrm{D}$ code CALE, ${ }^{14}$ and the simulations. This is followed by a description of the new two-mode interfaces and a discussion of model predictions. The simulation results are presented and shown to exhibit complex behavior that is beyond the reach of existing models. When the drive is sufficiently strong, the nonlinear evolution of the short-wavelength mode can lead to the formation of jets that strongly affect the large-scale structure of the interface. This effect represents a nonlinear coupling between spikes and between spikes and bubbles. We describe the process of jet formation and its impact on the late-time interface evolution, including a significant dependence on the relative phases of the two modes. We compare the simulation results with data from recent two-mode experiments
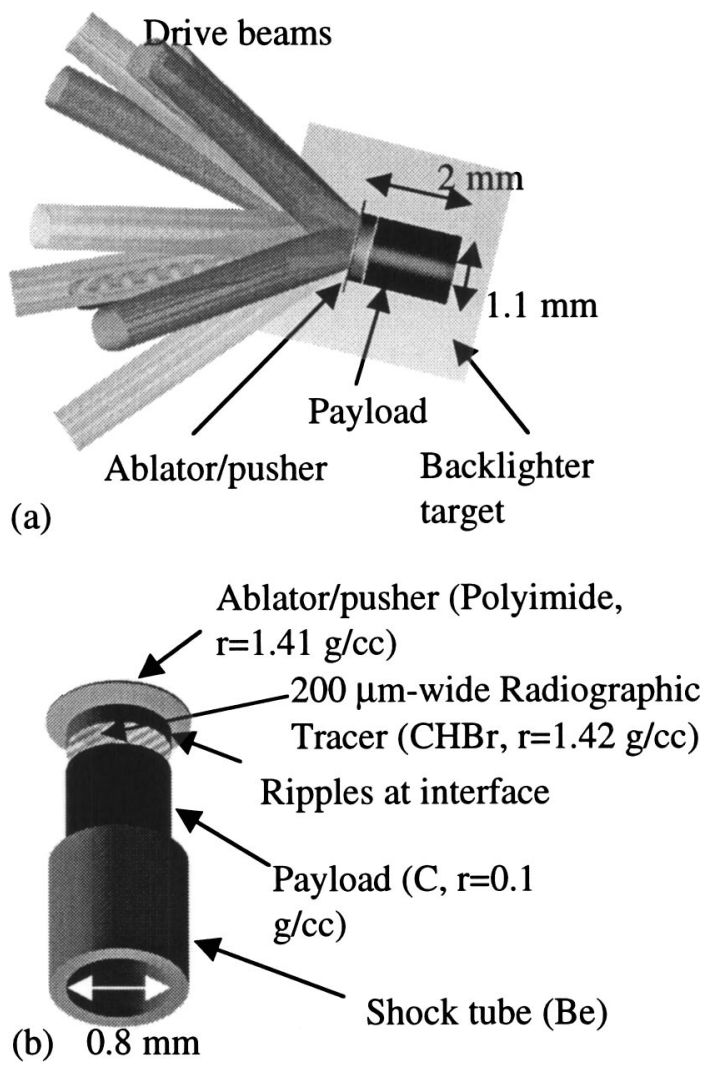

FIG. 1. Target schematic showing (a) the overall experiment configuration and (b) an exploded view of the target package.

and consider earlier few-mode experiments in the context of these effects. Finally, we conclude with a summary of our results and a brief outline of our current work with broadband initial conditions.

\section{EXPERIMENT}

The experiment (see schematic in Fig. 1), which is described in greater detail elsewhere, ${ }^{11}$ uses ten of the OMEGA laser's beams in a $5 \mathrm{~kJ} 1 \mathrm{~ns}$ pulse to drive a Mach 15 blast wave into one end of a cylindrical target. The average laser intensity on the target is typically $6 \times 10^{14} \mathrm{~W} / \mathrm{cm}^{2}$. The target consists of a heavier plastic pusher/ablator section [polyimide $\left(\mathrm{C}_{22} \mathrm{H}_{10} \mathrm{~N}_{2} \mathrm{O}_{4}\right)$ with a density of $\left.1.41 \mathrm{~g} / \mathrm{cc}\right]$ and a lighter foam payload section (carbonized resorcinol formaldehyde with density $0.05 \mathrm{~g} / \mathrm{cc}$ ) in contact with one another along a perturbed interface. In order to reduce lateral expansion, this multicomponent target assembly sits within a Be shock tube. Because the perturbation is machined into the plastic pusher but not into the foam payload, the two materials are actually in contact only at the highest peaks of the perturbation. As a result, there exists a gap between the two materials.

The interface velocity and deceleration (taken from a 1D CALE simulation) are plotted as functions of time in Fig. 2. As the shock front crosses the interface at $1 \mathrm{~ns}$ and impulsively accelerates it up to about $70 \mathrm{~km} / \mathrm{s}$, it deposits vorticity, which drives RM growth. The interface then begins to decelerate, and does so for the 40 ns remainder of the experiment. During the deceleration phase, the interface is RT un- 


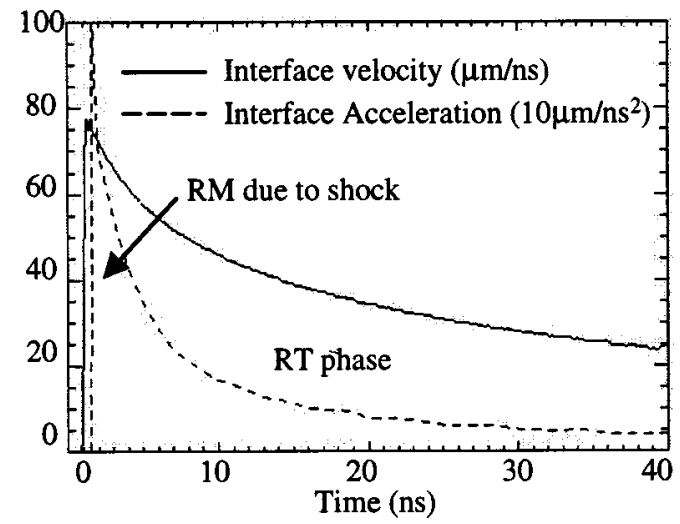

FIG. 2. Interface velocity and deceleration. The shock induces RM growth and the interface is RT unstable during the subsequent deceleration phase. The postshock Atwood number is nearly constant at $A^{*}=0.70$.

stable. Shock-deposited vorticity (RM) dominates the perturbation growth for the first couple of nanoseconds, while accereleration-induced (RT) growth dominates at later times. In addition to the RM and RT instabilities, target decompression occurs during the experiment, and is responsible for about $50 \%$ of the total perturbation growth at late times. ${ }^{15}$

The experiments are diagnosed via side-on and face-on $\mathrm{x}$-ray radiography throughout their duration of up to $40 \mathrm{~ns}$. This is done with titanium backlighters that produce $4.7 \mathrm{keV}$ photons for target imaging. A nearly mass-matched radiographic tracer is embedded in the plastic pusher in order to reduce edge effects and concentrate $\mathrm{x}$-ray opacity into a relatively thin slice near the target axis.

\section{SIMULATION}

The experiments are simulated using the 2D radiationhydrodynamics code CALE. CALE is an arbitrary Lagrangian-Eulerian (ALE) code that uses a second-order (in space and time) finite differencing method to numerically solve the Euler equations. ${ }^{14}$ The relative importance of several numerical and physical factors for achieving good agreement between simulation and experiment were considered in detail in an earlier paper. ${ }^{15}$ In accordance with those results, the simulations in this work are characterized as follows: The computational grid spans the length of the target, and its width is generally determined by the minimum allowable considering symmetry constraints, i.e., one-half wavelength for a single-mode simulation). The transverse resolution is 120 points per perturbation wavelength (ppw) of the longest wavelength $(50 \mu \mathrm{m})$ mode. The specified boundary conditions (BCs) are reflecting along the mean flow direction and free along the transverse direction. Simulations are run in Eulerian rather than ALE mode in order to avoid spurious $\mathrm{KH}$ activity at the evolving interface. The gap between the plastic and foam sections is included and filled with $1 \mathrm{mg} / \mathrm{cc}$ air or foam. Electron conduction is included, but the effects of x-ray and electron preheat are not. Tabular equations of state (EOS) are used for all materials, with LEOS or EOP for the plastic and EOP for the foam. Simulations are initialized with a driving slab with uniform temperature, density, and velocity taken from a 2D LASNEX (Ref. 16) calculation.

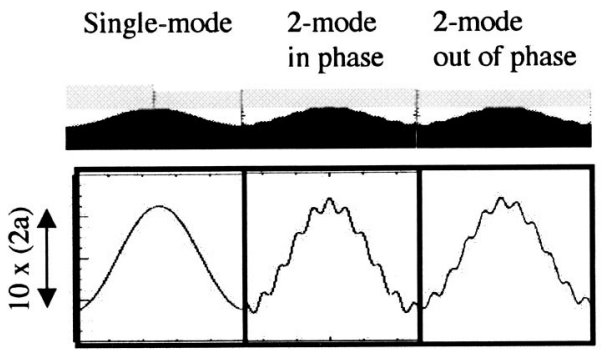

Mode 1: $\lambda=50 \mu \mathrm{m}, \mathrm{a}=2.5 \mu \mathrm{m}(\mathrm{ka}=0.3), 120 \mathrm{ppw}$ Mode 10: $\lambda=5 \mu \mathrm{m}, \mathrm{a}=0.25 \mu \mathrm{m}(\mathrm{ka}=0.3), 12 \mathrm{ppw}$

FIG. 3. Single-and two-mode initial interfaces. The short-wavelength mode (mode 10) is either in-phase or $\pi / 2$ out-of-phase with the long-wavelength mode (mode 1).

Such simulations have been shown to agree well, both in interface structure and spike-bubble averaged perturbation amplitude growth, with experiments involving single-, two-, and eight-mode interfaces. ${ }^{15}$ For the single-mode simulations, separate spike and bubble amplitude histories (as well as their average) were reasonably well predicted by a buoyancy-drag model when target decompression effects are first removed. Aside from the initial modal structure, the earlier experiments differ from those discussed in this work only in the foam density-now 50 rather than $100 \mathrm{mg} / \mathrm{cc}$.

\section{NEW TWO-MODE INTERFACE (SHORT ON LONG) WITH VARYING PHASE}

We now consider the same single-mode perturbation studied previously (with a $50 \mu \mathrm{m}$ wavelength and $2.5 \mu \mathrm{m}$ initial amplitude), and study the effect of a single shortwavelength component on its evolution. The scale of the secondary mode (mode 10) is one-tenth that of the primary mode (mode 1), or $5 \mu \mathrm{m}$ in wavelength and $0.25 \mu \mathrm{m}$ in initial amplitude (see Fig. 3). With 120 ppw in mode 1, mode 10 is resolved in the simulations to $12 \mathrm{ppw}$. According to Ofer et al. ${ }^{17}$ the finite numerical resolution of an RT-unstable mode (for incompressible flow under constant acceleration) results in a growth rate reduction below the theoretical value that is given by

$$
\gamma_{\text {eff }}=\gamma_{\text {theoretical }}(1-2 / \mathrm{ppw}) \text {. }
$$

For mode 10, Eq. (1) predicts a $17 \%$ reduction in growth rate relative to the fully resolved value.

Ofer $e t a l$. in their consideration of the effect of a secondary short wavelength on a primary long-wavelength mode, found that the main effect of the short-wavelength component was to introduce an effective density gradient that acted to stabilize the growth of the primary mode. ${ }^{18}$ They used a 2D ALE code in their study and, like us, considered a moderate Atwood number $(A=0.5$ compared to our post-shock $\left.A^{*}=0.7\right)$. Their calculations were slightly better resolved than ours, with 17-18 ppw in the shortest wavelength mode (compared to our value of $12 \mathrm{ppw}$ in the shortest wavelength mode). There are, however, several significant differences between their study and ours. Theirs was a pure RT system (no RM) with constant acceleration, while 
our blast-wave-driven interface is both RM and RT unstable with decaying acceleration. Our instability Mach number is higher, with $M=0.15-0.20$ in foam and $0.25-0.40$ in plastic, than their $M<0.1$. It is also significant that their longwavelength mode remains linear throughout, while both of our modes enter the nonlinear regime early on. Finally, their short-wavelength component is in-phase (or $180^{\circ}$ out-ofphase, depending on one's choice of the positive direction). In order to investigate the phase dependence of the instability in the deep nonlinear regime, the relative phase of mode 10 with respect to mode 1 is in our study either $0, \pi / 4$, or $\pi / 2$.

With a phase difference of 0 or $\pi$, the initial symmetry allows us to limit the computational domain to one-half of the mode-1 wavelength. Since CALE does not allow for periodic $\mathrm{BCs}$, a reasonable treatment of the out-of-phase case requires that we include multiple wavelengths. This requirement must be balanced with the need to run with reasonably high resolution and in a reasonable amount of CPU time. In light of these considerations, we include four mode-1 wavelengths in the out-of-phase case. The two modes are then technically modes 4 and 40, but because the ratio of their wavelengths is an integer, no modes lower than 4 can be generated via mode coupling. Consequently, the system is equivalent to the mode-1/mode-10 system in the region acoustically isolated from the boundaries (while such a region exists). Late time density plots from the out-of-phase simulation show some competition between mode- 4 bubbles, but not merger and the associated generation of larger, faster growing structures.

\section{MODEL PREDICTIONS}

In considering model predictions, we divide the perturbation evolution into three regimes: linear, early nonlinear, and nonlinear. Simple theory predicts that, neglecting certain potentially stabilizing factors such as surface tension, ${ }^{19}$ each RT-unstable mode grows exponentially in the linear regime $(a / \lambda<0.1)$ with a growth rate given by ${ }^{20}$

$$
\gamma=\sqrt{\frac{k g A}{1+k L}},
$$

where $k$ is the perturbation wave number $2 \pi / \lambda, g$ is the (constant) acceleration, $A$ is the Atwood number, and $L$ is the density gradient scale length at the interface. Thus the RT exponential growth rate $(1 / a) d a / d t$ for mode 10 is greater than for mode 1 by a factor of $\sqrt{10}$ (i.e., $\gamma_{\text {mode } 10}$ $\left.=\sqrt{10} \gamma_{\text {mode } 1}\right)$. The amplitude growth rate $d a / d t$ of mode 10 is smaller than for mode 1 by the same factor $\left(d a / d t_{\text {mode }} 10\right.$ $\left.=d a / d t_{\text {mode }} / \sqrt{10}\right)$.

According to Richtmyer's impulsive RM model ${ }^{4}$ adapted by Meyer and Blewett for the heavy to light case, ${ }^{21}$ each RM-unstable mode amplitude grows linearly in time during the linear phase at the Meyer-Blewett velocity, given by

$$
v=k a_{0} A^{*} u_{i} \text {. }
$$

Here $a_{0}$ is the average of the preshock and postshock perturbation amplitudes, $A^{*}$ is the postshock Atwood number, and $u_{i}$ is the velocity increase of the interface upon shock transmission. Because both the amplitude and wavelength of the short-wavelength mode are scaled in the same way relative to long-wavelength mode, the Meyer-Blewett velocity is the same for modes 1 and 10 .

According to linear modal analysis where modes grow independently without interacting, mode 10 can have no effect on mode 1 regardless of the relative phase between the two modes. Ofer et al. found that the short-wavelength mode does introduce an effective density gradient at the interface that will somewhat stabilize the primary mode [via $L$ in Eq. (2)]. ${ }^{18}$ According to their analysis, the mode-1 linear RT growth rate is reduced by $10 \%$ when $(a / \lambda)_{10}$ reaches about 0.3 , or about the time mode 10 reaches its saturation velocity.

Because the targets under consideration are driven very strongly and the initial amplitudes are somewhat large (preshock $a / \lambda \approx 0.05$ and postshock $a^{*} / \lambda \approx 0.02$ ), the linear approximation is valid for a very short period of time. Considering RT only, modes 1 and 10 reach the nonlinear threshold value of $a / \lambda=0.1$ in about $2 \tau^{\mathrm{RT}} \approx 2.0$ and $2 / 3 \mathrm{~ns}$, respectively. For RM only, the linear approximation breaks down in about $\tau_{\mathrm{nl}}^{\mathrm{RM}} \approx 0.5$ and $0.05 \mathrm{~ns}$ for mode 1 and mode 10 , respectively. Thus RM growth, which dominates for the first couple of nanoseconds provides the stronger limit on the linear regime. Mode 1 becomes nonlinear within $1 \mathrm{~ns}$ (of a $40 \mathrm{~ns}$ experiment) and mode 10 is nonlinear virtually instantaneously.

At later times, initially sinusoidal perturbations grow into characteristic spikes and bubbles. In the early nonlinear phase, which is also very short, mode coupling is present but weak, and Haan's spectral model ${ }^{22}$ is valid. Harmonic generation (of modes 2 and 20) introduces spike-bubble asymmetry, with spikes growing faster than bubbles. In addition, modes 1 and 10 couple to generate modes 9 and 11 .

The saturation velocity of the primary mode can in principle be reduced by the presence of a second mode where the two interfere constructively. ${ }^{23}$ Mode 10 interferes constructively with mode 1 at each mode- 1 bubble tip when the two are in phase. However, because of their large separation in wave number space, both modes should saturate at the single-mode terminal velocity. Modes subsequently generated by mode coupling should also not reduce the mode-1 saturation time for the same reason and also because of how quickly mode 1 becomes nonlinear.

The early nonlinear phase ends when third order terms (and soon thereafter terms of all order) in the perturbation expansion become significant and the bubbles approach terminal velocity. For constant acceleration RT with $A=1$, the bubbles rise with constant (terminal) velocity while spikes fall with constant acceleration in the nonlinear regime. ${ }^{24,25}$ When $A<1$, the spike also reaches terminal velocity. ${ }^{25}$

Mode coupling between modes 1 and 10 is fairly weak (third order). In addition, Ofer et al. ${ }^{17}$ found that once a mode has reached its saturation amplitude, it no longer contributes to the growth of longer wavelength modes. Since mode 10 becomes nonlinear in well under a nanosecond, it does not have time to significantly affect the interface evolution via mode coupling. During the nonlinear phase, bubbles (and spikes for $A<1$ ) grow at terminal velocity $\propto \sqrt{\lambda}$, where $\lambda$ is the object's transverse size. ${ }^{24}$ Since larger structures grow faster, an inverse cascade driven by bubble 
competition and merger sets in and washes small-scale bubbles downstream. ${ }^{26,27}$

The coupling strength in modal models ${ }^{22,17}$ depends on the relative phases of interacting modes according to $\cos \left(\varphi_{j} \pm \varphi_{k}-\varphi_{i}\right)$ for mode $i$ driven by the interaction of modes $j$ and $k$. The plus sign is mode $i$ generated by shorter wavelength modes (harmonic generation) while the minus sign is for mode $i$ generated by the interaction of longer wavelength modes. Thus the coupling increases with increasing phase coherence and vanishes for modes that are out-ofphase.

The late-time scaling is determined by the nature of the time-dependent acceleration. If $g(t)$ falls off slower than $t^{-2}$, then the instability growth is RT rather than RM-like. ${ }^{28}$ For an $n$-dimensional blast wave in the self-similar regime, dimensional analysis requires that the shock-front deceleration scales like $t^{-2(n+1)(n+2)}$. Away from the shock front, the resulting deceleration of the driven interface scales like $t^{-2(1-1 /[\gamma(n+2)])}$, where $\gamma$ is the adiabatic index. In the infinitely compressible case $(\gamma=1)$, the exponent is equal to $-4 / 3$ when $n=1$ and is greater than -2 for all positive $n$. For any dimension $n$, the exponent approaches -2 from above as the adiabatic index becomes large. Consequently, neglecting the true RM component associated with the passage of the shock front, perturbation growth at a blast-wave-driven interface is always RT-like. The bubble distribution approaches a scaleinvariant attractor and then the growth of the bubble and spike fronts scales like ${ }^{29,30} h_{s, b} \sim \alpha_{s, b} A\left[\int d t \sqrt{g}\right]^{2}$ or $h_{s, b}$ $\sim \alpha_{s, b} A t^{2 /(3 \gamma)}$ for a 1D blast wave (neglecting material decompression). This follows from the assumption that the height of the front is proportional to the dominant wavelength or transverse bubble size and gives the well-known quadratic dependence for constant acceleration. ${ }^{31}$ In experiments and simulations, however, the inverse cascade to successively larger structures is limited by the size of the physical or computational box. In our simulations, which are intended to study the effect of a high 1-mode on a dominant low 1-mode, the box size is not large compared to the wavelength of the lowest 1-mode with significant initial amplitude (mode 1 for the single-mode and in-phase cases and mode 4 for the out-of-phase case). Once mode 1 becomes the fastest growing mode (which is true very early on due to its large initial amplitude) a scale-invariant bubble distribution cannot be attained. If we therefore assume that the dominant wavelength remains constant and the acceleration falls off like $t^{-2[1-1 /(3 \gamma)]}$, then we find that $h \sim t^{1 /(3 \gamma)}$. This asymptotic behavior is captured by buoyancy-drag models such as that of Oron et al. where the spike (s) and bubble (b) evolution are governed by the equations ${ }^{32}$

$$
\left(\rho_{b, s}+2 \rho_{s, b}\right) \frac{d u_{b, s}(t)}{d t}=\left(\rho_{s, b}-2 \rho_{b, s}\right) g(t)-\frac{6 \pi}{\lambda} \rho_{s, b} u_{b, s}^{2} .
$$

In order to make some prediction of the late-time dependence on the initial phases, we have applied the modal model of Ofer et al. ${ }^{17}$ with some modifications. In the early nonlinear stage, mode growth and coupling are determined by Haan's weakly nonlinear model. ${ }^{22}$ Unlike Haan's model, modes generated by mode coupling can couple with each

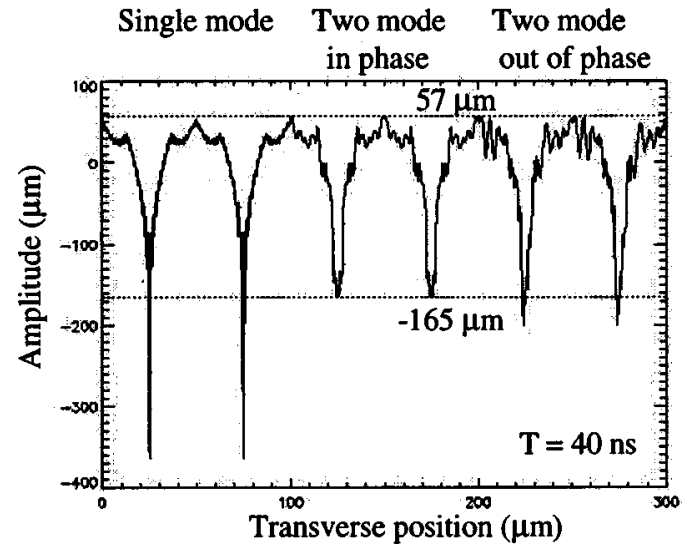

FIG. 4. Modal model prediction of interface at $40 \mathrm{~ns}$. The upper dotted line denotes the bubble position $(57 \mu \mathrm{m})$, which is approximately equal for all three cases. The lower dotted line (at $-165 \mu \mathrm{m}$ ) shows the approximate position at which the predicted spike widths fall below the minimum value observed in the simulations (about $2 \mu \mathrm{m}$ ). Like the predicted bubble amplitude, the spike amplitude defined in this way is virtually the same for all three cases. The Kelvin-Helmholtz instability prevents the spikes from attaining shapes predicted by the model. The model also does not include material decompression and RM contribution.

other and with preexisting modes. This amounts to an approximate inclusion of higher order terms, thereby extending the model's range of validity. Modes saturate according to the Haan criterion ${ }^{23}$ and subsequently do not contribute to the growth of lower 1-modes. Saturated low 1-modes can, however, modify the phases of higher 1-modes through harmonic generation as long as the velocity of the driven mode does not exceed its saturation value. In our application of the model, the time dependence of the acceleration is included. In addition, our treatment of phases is more general than in the original implementation, which effectively allowed for cosine modes with phases of only 0 and $\pi$. In our case, the coupling term includes the $\cos \left(\varphi_{j} \pm \varphi_{k}-\varphi_{i}\right)$ term. When the interaction of two modes generates a new mode, the phase of the new mode is determined by the resonance condition $\varphi_{i}$ $=\varphi_{j} \pm \varphi_{k}$. When an existing mode $i$ is driven by two modes $j$ and $k$, producing a velocity increment $d v_{i}$, the phase of the driven mode is shifted according to

$$
\varphi_{i}^{n}=\tan ^{-1}\left|\frac{a_{i}^{n-1} \sin \left(\varphi_{i}^{n-1}\right)+d v_{i} d t \sin \left(\varphi_{j} \pm \varphi_{k}\right)}{a_{i}^{n-1} \cos \left(\varphi_{i}^{n-1}\right)+d v_{i} d t \cos \left(\varphi_{j} \pm \varphi_{k}\right)}\right|,
$$

where $n$ is the time step and $a_{i}$ is the amplitude of mode $i$. This follows from the requirement that $a_{i} \cos \left(k_{i} x+\varphi_{i}^{n}\right)$ $=a_{i}^{n-1} \cos \left(k_{i} x+\varphi_{i}^{n-1}\right)+d v_{i} d t \cos \left[\left(k_{j} \pm k_{k}\right) x+\varphi_{j} \pm \varphi_{k}\right]$ since, by definition, $k_{i}=k_{j} \pm k_{k}$.

The result is shown in Fig. 4. For the bubble amplitude, the model predicts essentially no late-time dependence of the large-scale structure on the initial presence of mode 10, let alone on its phase relative to that of mode 1 . There is a strong effect predicted for the spike amplitude. However, since the spike evolution will clearly be strongly affected by $\mathrm{KH}$ rollup long before it reaches the shape predicted by the modal model, this prediction should not be taken too seriously. In fact, if we define the spike position as the point at which the spike width falls below $2 \mu \mathrm{m}$ (approximately 


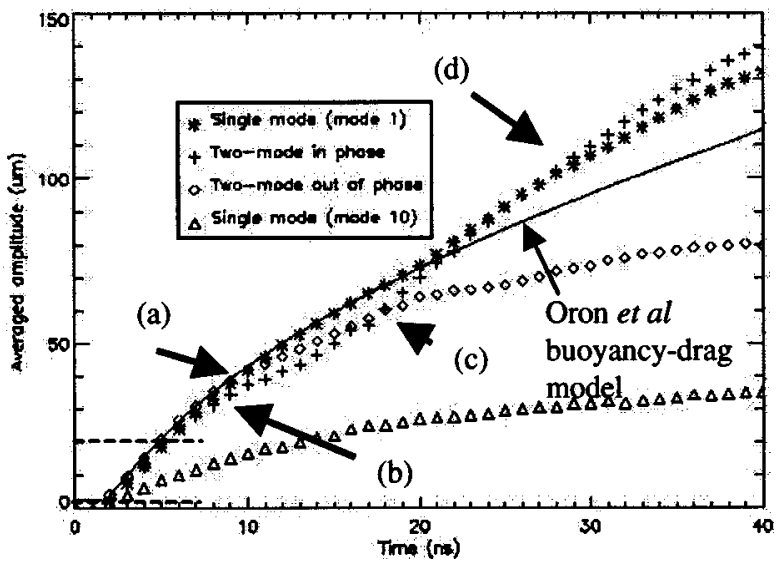

FIG. 5. Spike-bubble averaged amplitudes corrected for decompression. (a) The bubble-merger-driven inverse cascade is complete by about $10 \mathrm{~ns}$. (b) Early on, the growth is not strongly affected by short- $\lambda$ "noise." (c) For the two-mode cases, sudden changes in growth rate occur at intermediate times. (d) After $20 \mathrm{~ns}$, the phase-correlated (decorrelated) noise leads to growth enhancement (suppression) relative to single mode. The dashed lines show the single-mode saturation values $(\mathrm{a} / \lambda=0.4)$ for modes 1 (upper) and 10 (lower).

equal to the width of four computational cells and the minimum spike width observed in the simulations), then the latetime effect of mode 10 on the spike amplitude virtually disappears.

In conclusion, the models considered predict that the effect of mode 10 on mode 1 via mode coupling will be weak at all times. Consequently, the main effect of mode 10 on the evolution of mode 1 should be a reduction of the growth of mode 1 due the effective density gradient provided by mode 10 .

There are several other factors not discussed here that could in principle play a role in the interface evolution. Of these, we are particularly interested in the late-time effect of the RM component, material decompression (particularly the resulting vortex dynamics in experiments that are best only quasi-2D), the relative contributions of electron conduction, viscosity, and finite resolution on stabilization of high-1 modes, reduction of Atwood number in the mixing layer due to the KH instability, and reduction in Atwood number at the spike and bubble tips due to the large-scale density gradient present behind the shock front. Some of these issues will be treated in a later paper in the context of broadband initial conditions.

\section{SIMULATION RESULTS}

The averaged amplitude histories for the different phase realizations (including mode 10 by itself) are shown in Fig. 5. Also included is the mode-1 prediction of the buoyancydrag model of Oron et al. [given in Eq. (4)]. ${ }^{32}$ In order to isolate the instability effects and facilitate comparison with the model, the effect of target decompression has been removed from all amplitude plots. ${ }^{15}$ As expected, the linear phase lasts no more than about $1 \mathrm{~ns}$. Before $10 \mathrm{~ns}$, the overall growth is not strongly affected by the presence of the short-wavelength mode. Consequently, the single-mode buoyancy-drag model provides a good description of the growth in all three cases. While the acceleration is large, mode 10 remains sufficiently small that the introduction of its amplitude into the buoyancy-drag model as a stabilizing density gradient results in virtually no change in the predicted mode- 1 growth.

At intermediate times (between 10 and $20 \mathrm{~ns}$ ), the multimode perturbation amplitude histories reveal abrupt changes in the growth rates. After first falling below the single-mode case, the growth of the in-phase interface suddenly increases, so that its final amplitude $(140 \mu \mathrm{m})$ is somewhat higher than in the single-mode case $(133 \mu \mathrm{m})$. The growth rate in the out-of-phase case also falls below the single-mode case at about $10 \mathrm{~ns}$. At about the time that the in-phase growth rate increases, however, the out-of-phase growth rate falls still further, so the averaged amplitude reaches only $80 \mu \mathrm{m}$ at $40 \mathrm{~ns}$. The result is that, after $20 \mathrm{~ns}$, the phase-correlated noise leads to some growth enhancement (by about $5 \%$ ) relative to the single mode while the phase decorrelated noise leads to tremendous growth suppression (by about $40 \%$ ).

Additional detail can be obtained from plots of separate spike and bubble amplitude histories, which are shown in Fig. 6. Without the short-wavelength component, the mode-1 amplitudes are as expected for an interface driven by a decaying acceleration. The spike and bubble growth rates fall off with the acceleration, and the amplitudes begin to saturate as the drive strength approaches zero, with the spike amplitude significantly larger than that of the bubble. The spike-bubble symmetry apparent in the mode- 10 simulation likely results from an effective low Atwood number due to the finite density gradient at the interface, which is significant compared to the early-time amplitude of mode 10 but not mode 1 . The two-mode in-phase case begins similarly, but the bubble growth rate suddenly increases at about $11 \mathrm{~ns}$. Late in time, the spike amplitude is somewhat reduced relative to the single-mode case (by less than $2 \%$ ), while the bubble shows tremendous growth enhancement (about 65\%). In fact the bubble amplitude is greater than the spike amplitude until about $25 \mathrm{~ns}$. The out-of-phase case strongly differs from both the in-phase and the single-mode cases. The bubble growth is again enhanced, though only slightly (by about $1 \%$ ), but there is tremendous reduction of the spike growth (by about 60\%). In contrast with the single-mode case, the spike and bubble growth are nearly symmetric in both of the two-mode cases.

In summary, mode 10 has little effect on mode 1 during the linear and early nonlinear phases of the instability evolution, but has a strong effect during the deep nonlinear phase when the driving acceleration has decayed to below $25 \%$ of its peak value. The effect can lead to either reduction or enhancement of the overall amplitude growth depending on the phase of mode 10 relative to mode 1 . In both cases, the short-wavelength noise has a symmetrizing effect on the spike-bubble growth. The phase-correlated noise causes some growth enhancement relative to the single-mode case while the phase-decorrelated noise results in tremendous growth reduction. Furthermore, this effect does not appear to result from mode 10 providing an effective density gradient at the mode-1 interface. 

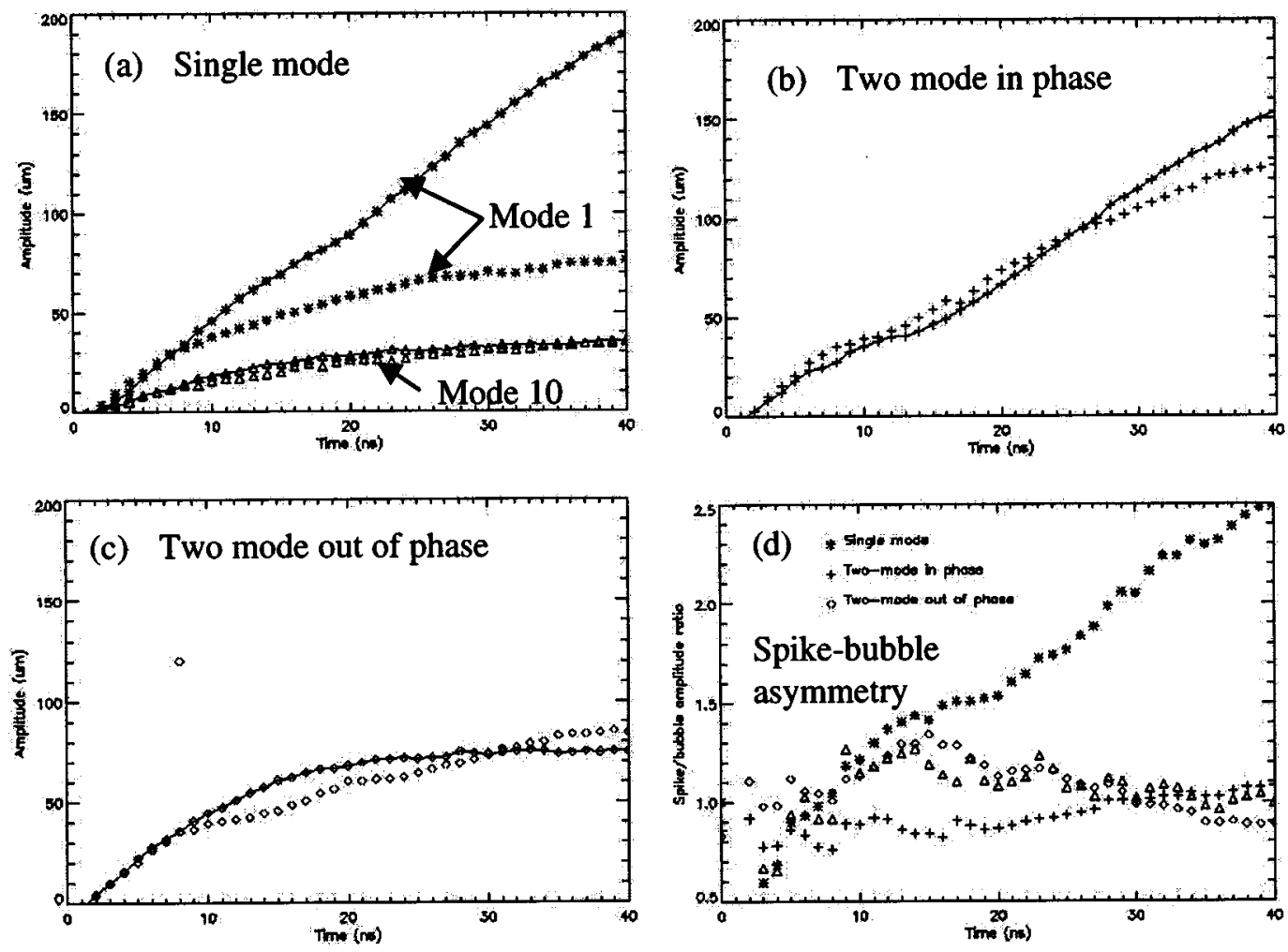

FIG. 6. Separate spike and bubble amplitudes corrected for decompression. In each case, the line denotes the spike amplitude. (a) The mode-1 single-mode growth is as expected, with the spike amplitude significantly larger than that of the bubble at late times. The spike and bubble growth is nearly symmetric in the mode-10 single-mode case because of the effective Atwood number reduction due to the density gradient at the interface. (b) In the two-mode in-phase case, there is some spike growth reduction relative to the single-mode case and tremendous bubble growth enhancement. (c) In the two-mode out-of-phase case, there is slight bubble growth enhancement and tremendous spike growth reduction. (d) A plot of the spike-bubble asymmetry (the ratio of spike to bubble amplitudes) shows nearly symmetric growth in two-mode cases.

The operative mechanism can be understood by observing the interface as it evolves (see Figs. 7-10). At 2 ns [1 ns after shock refraction - see Fig. 7(a)], both modes are apparent in the two-mode cases, and the effect of mode 10 on mode 1 is clearly small. At this point mode 1 is just entering the early nonlinear phase $(a / \lambda=0.1)$ while mode 10 has already attained $a / \lambda=0.4$. In the single-mode case, KH rollup at the spike tips is apparent within $3 \mathrm{~ns}$ of the interaction time. In the two-mode cases, the bubble merger process proceeds rapidly. The ten small-scale bubbles per mode- 1 wavelength present at $2 \mathrm{~ns}$ merge into five by $4 \mathrm{~ns}$, and then into two or three (depending on the phase) by $6 \mathrm{~ns}$. After one more merger, the process is complete by $10 \mathrm{~ns}$. By $5 \mathrm{~ns}$ [see Figs. 7(b) and 7(c)] the shape of the primary spikes has been significantly altered by the presence of mode 10 . The remaining secondary spikes (bubble merger is already underway) near the tips of the primary spikes have acquired a transverse growth component that is particularly pronounced in the inphase case [see Fig. 7(d)]. In the in-phase case, pairs of transversely growing secondary spikes collide with one another at about $8 \mathrm{~ns}$ [Fig. 8(a)], driving premature bubble merger and producing upstream- and downstream-directed jets. Since the collision direction is nearly perpendicular to the main flow direction, most of the collision energy is directed downstream. In the out-of-phase case, only a grazing collision occurs because every other of the secondary spikes (at the primary spike tips) still has a significant upstream velocity component. As a result, half of these secondary spikes are directed downward and eventually strike the primary spike stalks at about $11 \mathrm{~ns}$ [Fig. 8(c)]. This in turn causes a sudden reduction in the spike amplitude growth rate and leads to the large reduction in spike growth relative to the single-mode case observed at late times. Also at about $11 \mathrm{~ns}$, the downstream-directed jets produced in the in-phase case strike the inner surface of the primary bubble tips, thereby depositing energy that suddenly accelerates the bubble growth. At later times, KH activity near the primary spike tips effectively regenerates the smaller scales lost due to bubble merger. The process of secondary spike collision and jet formation can then continue, particularly in the inphase case. This occurs, for example, between 26 and $30 \mathrm{~ns}$ (see Fig. 9). Each new jet sends more spike material downstream into the primary bubble region, so that the coupling between $\mathrm{KH}$ and secondary spike interaction results in greatly enhanced mixing in the layer in addition to additional coupling between and generation of scales.

In the out-of-phase case, a large-scale vortex begins to form across several mode- 1 wavelengths between 26 and $30 \mathrm{~ns}$. This signifies that the edges of the computational domain have begun to influence the interface evolution all along the transverse direction. By this time, however, the spike amplitude in the out-of-phase case has already been greatly reduced relative to the other cases and has nearly saturated. 


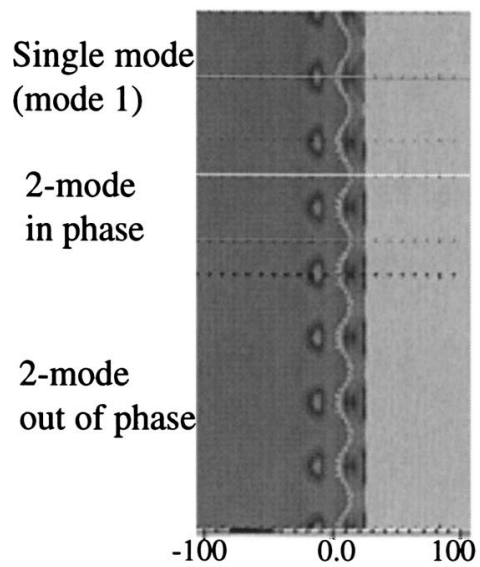

(a) $2 \mathrm{~ns}$
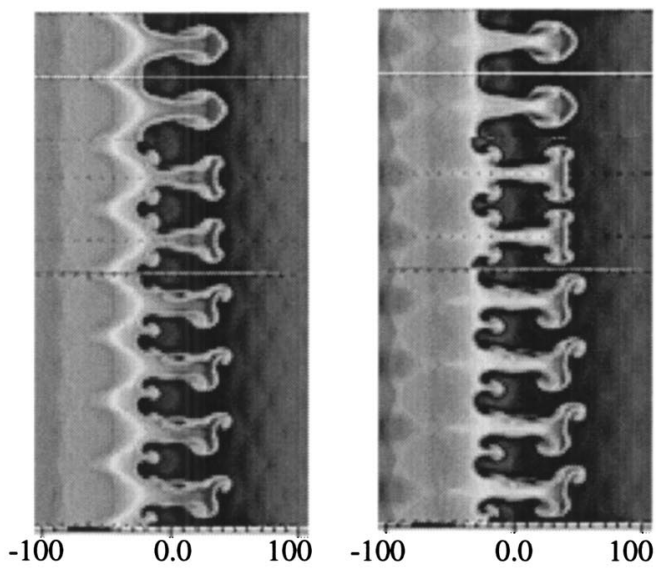

(c) $6 \mathrm{~ns}$

(d) $7 \mathrm{~ns}$

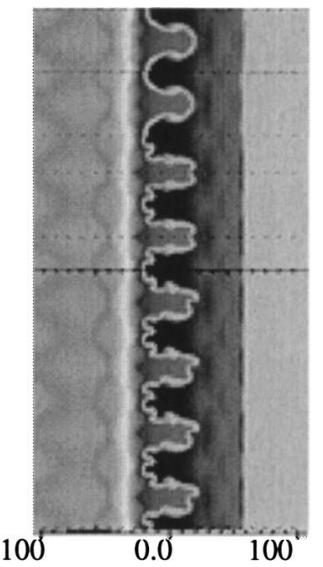

(b) $4 \mathrm{~ns}$

100
FIG. 7. Density plots at (a) $2 \mathrm{~ns}$, (b) $4 \mathrm{~ns}$, (c) $6 \mathrm{~ns}$, and (d) $7 \mathrm{~ns}$. At $M>0.1$, the drag force affects not only the saturation velocity, but also the shape of the spike and bubble.

At very late times, there are large differences in the interface structure of all three similations (see Fig. 10). Thus the large-scale features present during the late nonlinear instability evolution are strongly affected by the details of the initial conditions. Not only the presence of the shortwavelength mode, but also its phase, has a dramatic impact on the final state.

\section{COMPARISON WITH EXPERIMENTAL DATA}

Two sets of two-mode short on long experiments have been performed on OMEGA to investigate the effect of a short-wavelength secondary mode on the evolution of a long-wavelength primary mode. The wavelengths and initial amplitudes of the two modes are the same as in the simulations. The first series of four targets was shot in April 2003. In an effort to obtain a true side-by-side comparison unaffected by shot-to-shot variations in drive energy, the interface in each of these targets was divided in two regionssingle mode on one side and two-mode on the other. Unfortunately, all information on which side of each target was single mode and which was two-mode was lost. Figure

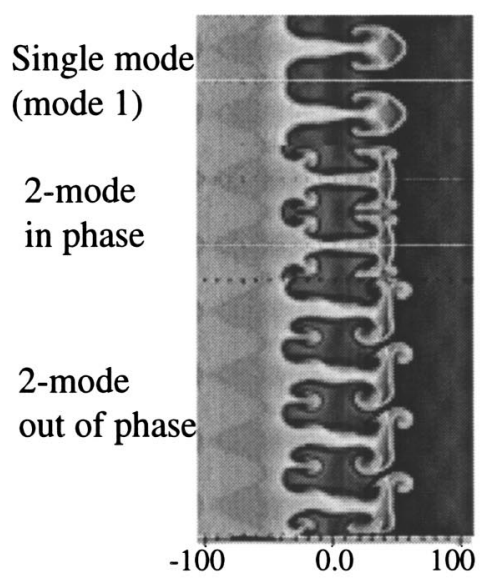

(a) $8 \mathrm{~ns}$

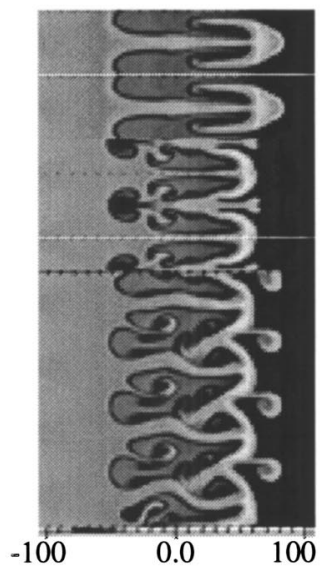

(c) $11 \mathrm{~ns}$

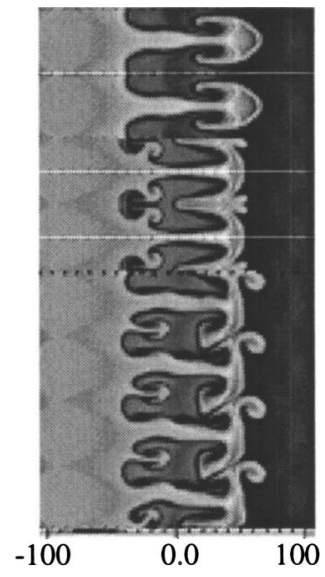

(b) $9 \mathrm{~ns}$

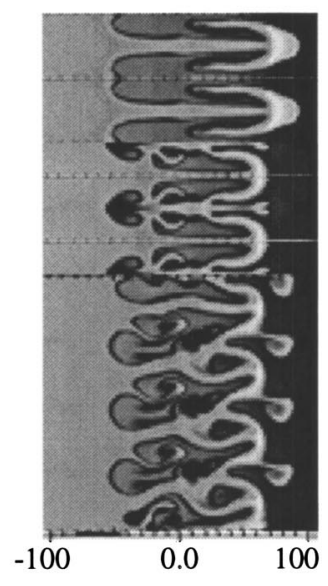

(d) $12 \mathrm{~ns}$
FIG. 8. Density plots at (a) $8 \mathrm{~ns}$, (b) $9 \mathrm{~ns}$, (c) $11 \mathrm{~ns}$, and (d) $12 \mathrm{~ns}$. Deflection of spikes results in colliding spikes, especially for interfaces consisting of periodic arrays of spikes (phase coherence). Fast colliding spikes drive premature bubble merger and produce upwards- and downwards-directed jets, with most of the energy directed down for collision perpendicular to the zero-order flow velocity direction. Downwards-directed jets strike the inner surface of bubble tips, thereby depositing energy that accelerates bubble growth.

11(a) shows a radiograph from one of these shots (half single-mode/half two-mode in-phase) at $25 \mathrm{~ns}$. The spikes corresponding to the long-wavelength mode are just beginning to break up all along the interface. Interpretation of the data is difficult because the single-mode side of the target may have been corrupted by spike breakup on the two-mode side or by the discontinuity at the boundary between the two sides. In addition, the discontinuity at the boundary makes observations near the centerline unreliable, and it is precisely this region that is least affected by interface curvature and therefore generally the source of the best data.

Because of these factors, the second target series (shot in August 2003) included two single-mode targets (imaged at 18 and $25 \mathrm{~ns}$ ) and two two-mode out-of-phase targets (at 25 and $30 \mathrm{~ns}$ ). Data obtained at $25 \mathrm{~ns}$ are shown in Fig. 11(b) and 11(c) to provide a comparison between the single-mode and the two-mode cases. The single-mode spike and bubble structure initially present in the single-mode target has per- 

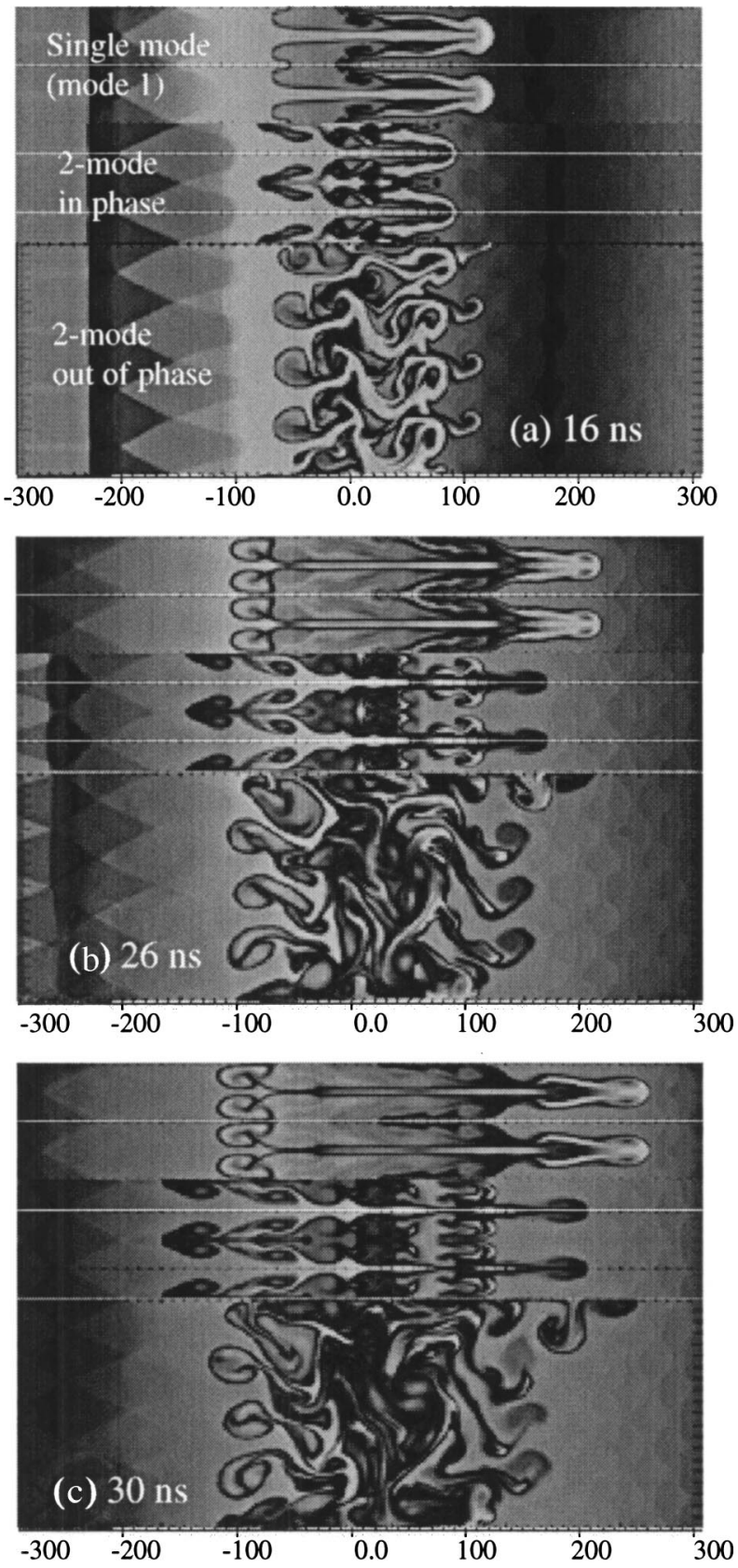

FIG. 9. Density plots at (a) $16 \mathrm{~ns}$, (b) $26 \mathrm{~ns}$, and (c) $30 \mathrm{~ns}$. Coupling of the spike interaction process with the $\mathrm{KH}$ instability results in additional coupling between and generation of scales and greatly enhanced mixing in the layer. This is a complicated nonlinear transfer of energy from spikes to bubbles and from transverse to parallel kinetic energy.

sisted to late times, while any such structure initially present in the two-mode out-of-phase target has vanished. As in the simulation, the presence of the short-wavelength mode appears to have completely broken up the primary mode. The uniformity of the x-ray transmission through the mix layer suggests that $3 \mathrm{D}$ structure has emerged and a transition to turbulence may have taken place.

Mix width data from all of the shots are compared in Fig. 12 with the simulation results, in this case shown without subtraction of the decompression effect. The data points are shifted by $1 \mathrm{~ns}$ to account for the way in which the simulations are initiated, and the experimental error is given by
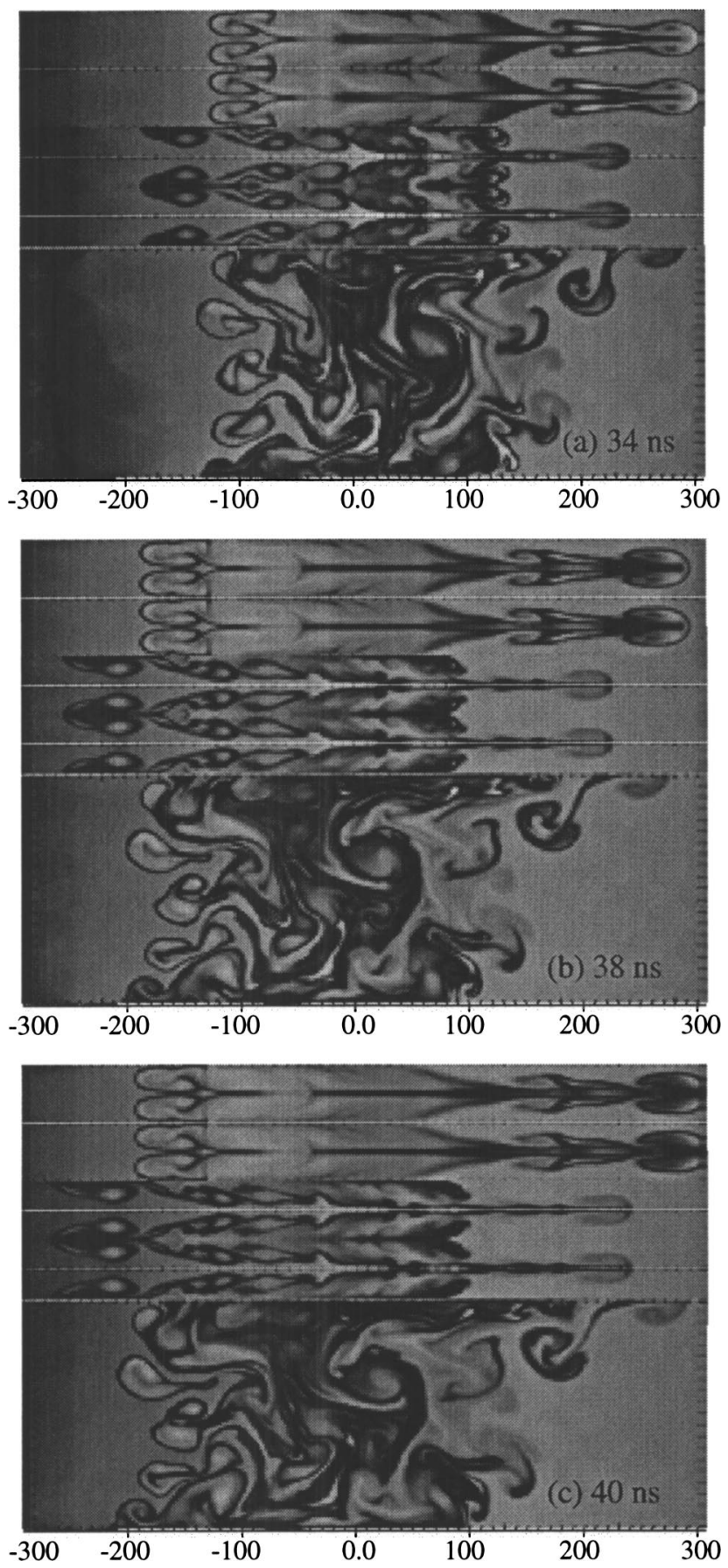

FIG. 10. Density plots at (a) $34 \mathrm{~ns}$, (b) $38 \mathrm{~ns}$, and (c) $40 \mathrm{~ns}$. The result is that large-scale features present during late nonlinear instability evolution are strongly affected by small-scale details of the initial conditions. In this case, phase coherence results in increased bubble growth while phase decoherence results in spike growth suppression.

the height of the data-point boxes. Two data points are included for each half single-mode/half two-mode-one for each side of the target. Below $20 \mathrm{~ns}$, all three simulations agree to within the experimental error, and the data agree with the simulations. The single-mode and in-phase calculations agree with each other above $20 \mathrm{~ns}$ as well, while the predicted out-of-phase amplitude falls significantly lower. Data are available for all three cases at $25 \mathrm{~ns}$, but the predicted amplitude reduction is still comparable to the mea- 


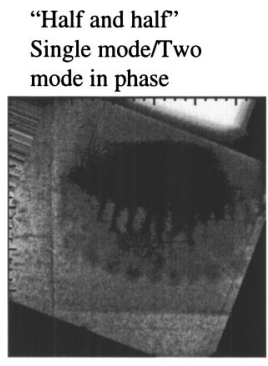

(a)
Apr. 25, 2003
Single mode

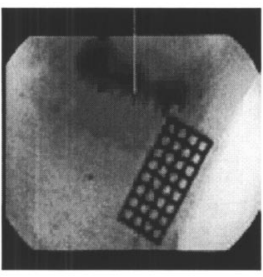

Signal $/$ noise $=1.146$
Two mode out of phase

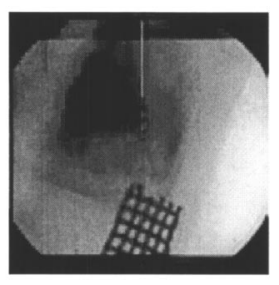

Aug. 21, 2003

(c)
FIG. 11. Experimental radiographs at $25 \mathrm{~ns}$. (a) A "half and half" target with a single-mode perturbation on one side and two-mode in-phase on the other exhibits single-mode-like spike and bubble structure that is just beginning to break down. (b) The single-mode target retains single-mode-like spike and bubble structure, while (c) the two-mode out-of-phase shows reduced growth and a breakdown of large-scale structures.

surement uncertainty. Above $25 \mathrm{~ns}$, available data are limited to an out-of-phase point at $30 \mathrm{~ns}$, which agrees with the simulation, and two points from a half single-mode/half outof-phase target at $37 \mathrm{~ns}$. The smaller-amplitude side of the target agrees well with the two-mode out-of-phase simulation, while the larger-amplitude side falls between the predicted single-mode and out-of-phase amplitudes. A possible explanation is that the larger-amplitude side is the singlemode perturbation corrupted by the out-of-phase side.

Though the quality and quantity of the data are not sufficient to definitively validate the simulation results, they do confirm that the presence of the secondary mode can dramatically alter the evolution of the mix region in the late nonlinear regime. They also suggest that the breakup of the large-scale spikes, which almost certainly indicates the presence of 3D flow and might correspond to a transition to turbulence, results in a large reduction in the growth of the

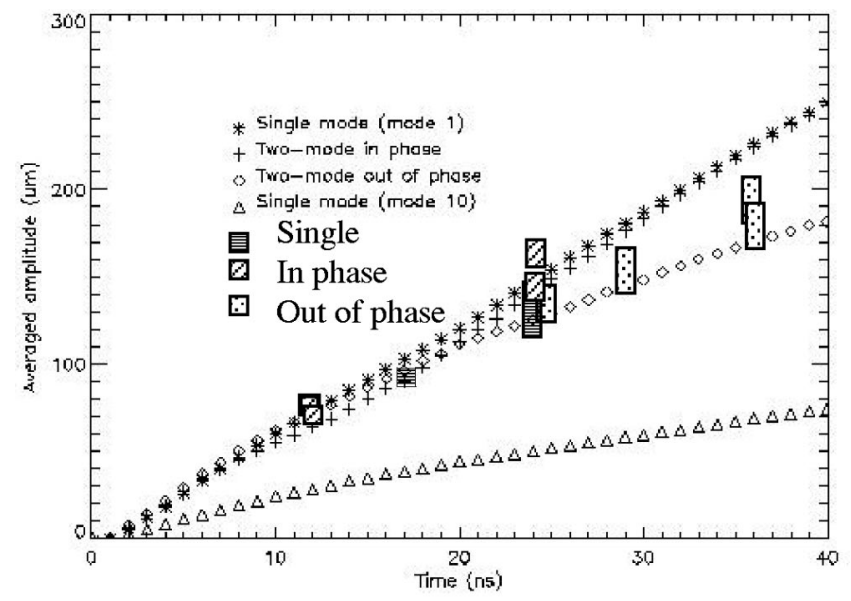

FIG. 12. Comparison of data with simulation without decompression effect subtraction. The size of the data points is the size of the error bars. Two data points are included for each "half and half" targets, one from the singlemode side and one from the two-mode side. Since it is uncertain which side is which, both points are labeled either in-phase or out-of-phase. As predicted by the simulations, the two, mode out-of-phase targets exhibit reduced growth relative to the single mode. The late-time behavior of the two-mode-in phase case cannot be resolved by the data.
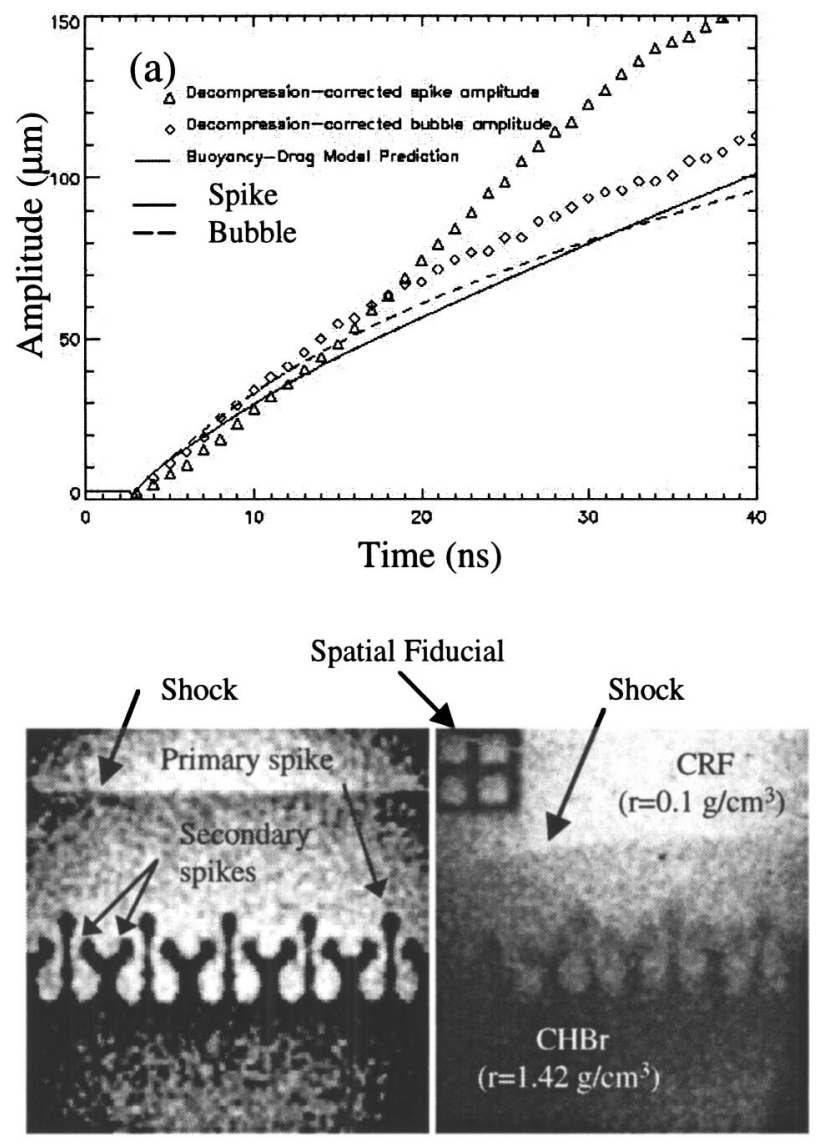

Simulated Radiograph

Experimental Radiograph

(b)

$$
\mathrm{T}=13 \mathrm{~ns}
$$

FIG. 13. Previous two-mode simulation and experiment show spike growth enhancement due to the interaction of secondary spikes. (a) Decompressioncorrected spike and bubble amplitudes. The spike velocity increases suddenly at about $15 \mathrm{~ns}$. (b) Simulated and experimental radiographs at $13 \mathrm{~ns}$, just prior to secondary spike interaction.

mix region relative to the single-mode result. In this case, the reduced amplitude is close to the prediction of the two-mode out-of-phase simulation.

\section{ANALYSIS OF EARLIER RESULTS}

In a previous paper, ${ }^{15}$ we presented simulations of twoand eight-mode experiments performed on the OMEGA laser. The two-mode perturbation [see Fig. 13(a)] was characterized by a sum of two sinusoidal components (in phase) with wavelengths of 40 and $60 \mu \mathrm{m}$ and amplitudes of 1.25 and $1.5 \mu \mathrm{m}$, respectively. The wavelengths present in the eight-mode case are given by $\lambda_{i}=180 \mu \mathrm{m} / i$ with $i$ ranging from 1 to 8 , while the amplitudes range from 0.4 to $0.7 \mu \mathrm{m}$. As in the two-mode case, all modes in the eight-mode case were in-phase. We found that CALE simulations agreed well with the experimental radiography in perturbation amplitude and interface structure. However, despite efforts to account for bubble merger, we were unable to successfully apply the buoyancy-drag model that had successfully predicted the single-mode growth. 


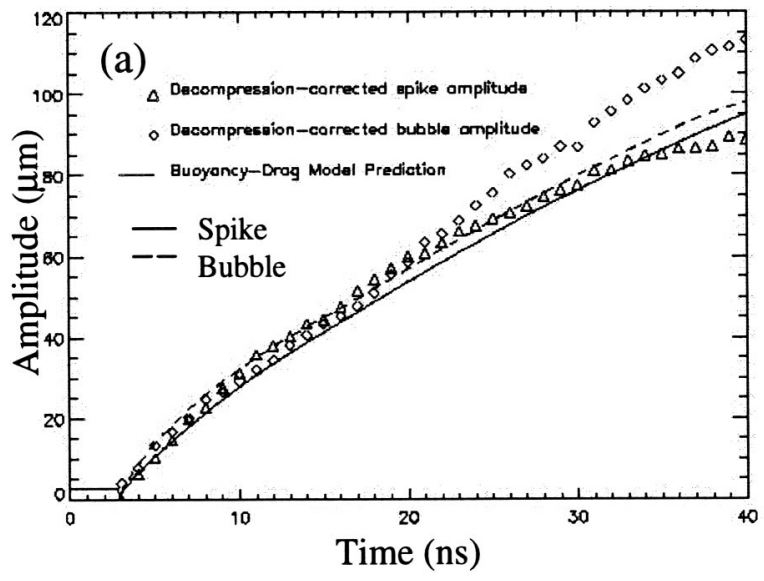

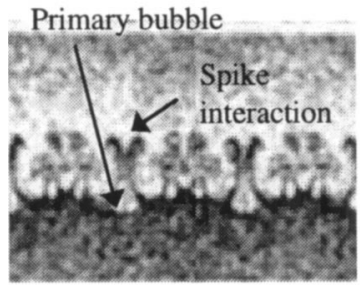

Simulated Radiograph

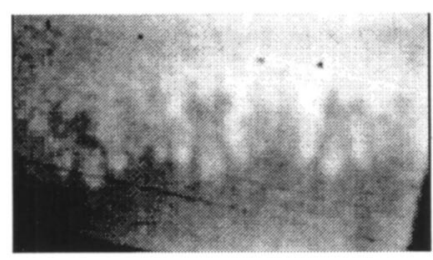

Experimental Radiograph (b)

$$
\mathrm{T}=13 \mathrm{~ns}
$$

FIG. 14. Previous eight-mode experiment and simulation show bubble growth enhancement due to jet production and interaction with bubbles (a) Decompression-corrected spike and bubble amplitudes. (b) Simulated and experimental radiographs at $13 \mathrm{~ns}$ showing spike interaction that leads to jet production above the primary bubble tip.

In the two-mode case, the bubble growth is consistent with the model description. The spike velocity, on the other hand, abruptly increases at about $15 \mathrm{~ns}$ and subsequently undergoes a period of growth that is nearly linear in time. This can now be understood in the context of the secondary spike interaction and jet formation process. A pair of secondary spikes with both transverse and upstream growth components is clearly visible near the tip of every other primary spike at $13 \mathrm{~ns}$ in both the simulation and experiment (see Fig. 13). In the simulation, the sudden increase in spike velocity occurs when these pairs of secondary spikes strike the intervening primary spikes, to which some of their upstream energy is transferred. Because the secondary spikes do not have a downstream growth component, there is no large downstream jet production and no consequent bubble growth enhancement.

In the eight-mode case, the collision of pairs of spikes (the distinction between "primary" and "secondary" is ambiguous because there is less separation of the initial modes in wave number space) does create downstream-directed jets that strike the inner bubble surface, resulting in an abrupt increase in bubble velocity at about $18 \mathrm{~ns}$ (see Fig. 14). At late times, the bubble amplitude is significantly greater (by about $30 \%$ ) than that of the spikes.

In both the two-and eight-mode cases, spike-spike interactions strongly influence the late-time amplitude histories. The exact nature of the effect, however, including the rela- tive influence on spikes and bubbles, depends on the details of the initial spectrum.

\section{DISCUSSION}

The aerodynamic drag pressure acting on the developing spikes not only determines their terminal velocity, but also affects their shape. As the instability growth rate increases, so too does the transverse velocity of redirected secondary spikes. The degree to which the large-scale interface structure is influenced when redirected secondary spikes collide with each other or with primary spikes is determined by their speed. The drag pressure is related to the thermal (or interface) pressure $P_{i}$ through the expression

$$
\rho_{s}^{*} v_{s}^{2}=\gamma_{s} M^{2} P_{i}
$$

where $M$ is the instability Mach number, $v_{s}$ is the spike velocity, and $\rho_{s}$ and $\gamma_{s}$ are the density and adiabatic index of the spike material. When the Mach number is equal to 0.3 ( $M^{2} \approx 0.1$ is the threshold for the appearance of compressibility effects ${ }^{33}$ ), the drag pressure is about one-tenth of the thermal pressure. Instability Mach numbers in our case in fact approach this regime (recall $M_{\text {foam }}=0.15-0.20$ and $\left.M_{\text {plastic }}=0.25-0.40\right)$. The process whereby the local pressure increases in secondary bubble regions results in partially redirecting the growth of secondary spikes into the transverse direction is reminiscent of the pinching effect noted by Li to occur during bubble merger. ${ }^{34}$ Because the flow velocity into the primary bubble is greater than into the secondary bubble, Bernoulli's equation requires that the pressure there be lower. Consequently, the spike dividing the two regions is redirected towards the primary bubble's center. Li hypothesized that the resulting reduction in effective Atwood number would eventually reduce the velocity of the larger bubble. But in our in-phase case, the head-on collision of two fastmoving secondary spikes produces an even faster downstream-directed jet. When it strikes the inner surface of the primary bubble, its ram pressure in the bubble frame is $0.45 \mathrm{Mbar}$-roughly equal to the $0.50 \mathrm{Mbar}$ thermal pressure of the plastic at the bubble position. It is therefore able to penetrate a significant distance into the plastic (in a process similar to a cratering event), thereby enhancing the bubble growth. Although there is no head-on collision of secondary spikes in the out-of-phase case, the redirected spikes are still sufficiently energetic to essentially punch through the primary spike stalks.

Whether or not redirected secondary spikes collide with each other or with primary spike stalks depends critically on the degree of phase coherence. Interfaces consisting of periodic arrays of spikes are more likely to evolve into colliding spikes. For a system such as ours with two commensurate modes, this requirement is satisfied when the modes are inphase. Of course, real physical systems such as interfaces within supernovae and ICF targets, whether characterized by only a few or by many modes, are very unlikely to exhibit high degrees of phase coherence. Indeed, the perfect symmetry enforced by the in-phase calculation is arguably rather unphysical. Systems comprised of incommensurate modes can, however, have regions of locally significant phase co- 
herence. Such regions will likely exhibit accelerated growth, but whether this eventually determines the dominant scales of the mix region remains to be seen.

In addition, there are important implications for those who wish to model physical systems. In multimode RT and RM simulations, the domain is often limited to a subsection or wedge of the full system with reflecting boundary conditions. In order to avoid unphysical effects at the boundaries, the initial perturbation spectrum sometimes includes only modes whose wavelengths are integer fractions of the full domain. The "random phase" assignment then amounts simply to a random assignment of plus or minus one to the amplitude of each mode. Since such spectra are actually characterized by a high degree of phase coherence, these simulations, if strongly driven, might significantly overpredict the growth of the mixing layer.

Regardless of the degree of phase coherence, the interaction of redirected spikes represents a coupling between transverse and parallel motions and a complicated nonlinear transfer of energy from spikes to other spikes (driving premature bubble merger) and to bubbles (perhaps resulting in increased spike-bubble symmetry). Coupling of this process with the KH instability results in additional coupling between and generation of scales and greatly enhanced mixing in the layer. Since turbulence requires the development of a broad inertial range of scales, this will likely decrease the time to transition.

The result that large-scale features present during the late nonlinear instability evolution are strongly affected by details of the initial conditions must be reconciled with the expectation that, at some point near or after transition, the mixing layer will begin to grow at a rate that is independent of the initial spectrum. If this expectation is correct, then the memory of the initial conditions must somehow be erased. The observed dependence would in that case be a transient phenomenon that would eventually disappear as the bubble size distribution settles into a scale invariant form. However, this argument requires the continual emergence of larger scales and depends on the existence of a sustained drive. In our case, the acceleration is decaying as the shock moves continually further away from the interface. At the same time, perturbation growth continues because of material decompression. The combination of a decaying drive and continuing decompression means that transients can effectively be "frozen in" to the flow and thereby persist to late times.

\section{CONCLUSIONS AND FUTURE DIRECTIONS}

In conclusion, we have shown one way in which compressibility affects the $2 \mathrm{D}$ evolution of hydrodynamically unstable interfaces. When the instability Mach number is higher than 0.1 , the pressure associated with kinematic drag on developing spikes can be sufficient to partially redirect spike growth into the transverse direction. For an interface with a long-wavelength primary mode and a shortwavelength secondary mode, the interaction of redirected secondary spikes introduces sensitivity to the initial conditions (both the spectrum and phase distribution). The developing instability then "remembers" small-scale details of the
ICs well into the late nonlinear stage of its evolution. Phase coherence tends to result in increased perturbation growthespecially of bubbles, which could lead some "random phase" multimode simulations to overstate the growth of the modeled physical system. Phase-decoherent noise generally results in growth suppression. Coupling of this process with the $\mathrm{KH}$ instability results in additional coupling between and generation of scales and greatly enhanced mixing in the layer.

Demonstrating the transition to turbulence in high Mach number experiments is a crucial step in developing an experiment-based understanding of supernova hydrodynamics. Nevertheless, in order to truly represent the desired astrophysical system, future experiments will need to not only reach transition, but to reach it early on while the acceleration is still large. This will likely require a significant increase in the drive intensity, and would also benefit from 3D ICs consisting of many short-wavelength modes. With this in mind, we are beginning to use adaptive mesh refinement simulations with NIF-like drive conditions to investigate the dependence on the spectrum and degree of phase coherence for interfaces with many modes over a wide range of scales. Finally, we note that we expect the jet effect to be significantly smaller, if not altogether absent, in 3D systems with broad spectra. Consequently, 3D calculations are being planned to study the effect of initial conditions on determining the time to transition and the properties of the subsequent turbulent flow.

\section{ACKNOWLEDGMENTS}

This work was performed under the auspices of the U.S. Department of Energy by the University of California, Lawrence Livermore National Laboratory under Contract No. W-7405-Eng-48. Work at the University of Michigan was supported by the U.S. Department of Energy under Grant Nos. DE-FG03-99DP00284, DE-FG03-00SF22021 and other grants and contracts.

${ }^{1}$ W. D. Arnett, J. N. Bachall, R. P. Kirshner, and S. E. Woosley, Annu. Rev. Astron. Astrophys. 27, 629 (1989).

${ }^{2}$ W. Hillebrandt and P. Hoflich, Rep. Prog. Phys. 52, 1421 (1989).

${ }^{3}$ R. Chevalier, Astrophys. J. 207, 872 (1976).

${ }^{4}$ R. D. Richtmyer, Commun. Pure Appl. Math. 13, 297 (1960).

${ }^{5}$ E. E. Meshkov, Izv. Akad. Nauk SSSR, Mekh. Zhidk. Gaza 4, 151 (1969).

${ }^{6}$ J. W. S. Rayleigh, Scientific Papers (Cambridge University Press, Cambridge, 1899).

${ }^{7}$ G. I. Taylor, Proc. R. Soc. London, Ser. A 201, 192 (1950).

${ }^{8}$ P. Amendt, J. D. Colvin, R. E. Tipton et al., Phys. Plasmas 9, 2221 (2002).

${ }^{9}$ D. Ryutov, R. P. Drake, J. Kane, E. Liang, B. A. Remington, and W. M. Wood-Vasey, Astrophys. J. 518, 821 (1999).

${ }^{10}$ P. E. Dimotakis, J. Fluid Mech. 409, 69 (2000).

${ }^{11}$ H. F. Robey, Y. Zhou, A. C. Buckingham, P. Keiter, B. A. Remington, and R. P. Drake, Phys. Plasmas 10, 614 (2003).

${ }^{12}$ B. Fryxell, E. Muller, and D. Arnett, Astrophys. J. 367, 619 (1991)

${ }^{13}$ J. M. Soures, R. L. McCrory, C. P. Verdon et al., Phys. Plasmas 5, 2108 (1996).

${ }^{14}$ R. T. Barton, Numerical Astrophysics (Jones and Bartlett, Boston, 1985).

${ }^{15}$ A. R. Miles, D. G. Braun, M. J. Edwards, H. F. Robey, R. P. Drake, and D. R. Liebrandt, Phys. Plasmas 11, 3631 (2004).

${ }^{16}$ G. B. Zimmerman and W. L. Kruer, Comments Plasma Phys. Controlled Fusion 2, 51 (1975)

${ }^{17}$ D. Ofer, U. Alon, D. Shvarts, R. L. McCroy, and C. P. Verdon, Phys. Plasmas 3, 3073 (1996).

${ }^{18}$ D. Ofer, D. Shvarts, Z. Zinamon, and S. A. Orszag, Phys. Fluids B 4, 
3549 (1992).

${ }^{19} \mathrm{~S}$. Chandrasekhar, Hydrodynamic and Hydromagnetic Stability (Dover Publications, New York, 1981).

${ }^{20}$ J. Lindl, Inertial Confinement Fusion: The Quest for Ignition and HighEnergy Gain (Springer, New York, 1998).

${ }^{21}$ K. A. Meyer and P. J. Blewett, Phys. Fluids 15, 753 (1972).

${ }^{22}$ S. W. Haan, Phys. Fluids B 3, 2349 (1991).

${ }^{23}$ S. W. Haan, Phys. Rev. A 39, 5812 (1989).

${ }^{24}$ D. Shvarts, O. Sadot, D. Oron, A. Rikanati, and U. Alon, in Handbook of Shock Waves, edited by G. Ben-Dor, O. Igra, and T. Elperin (Academic, London, 2001), Vol. 2, Chap. 14, pp. 489-543.

${ }^{25}$ H. J. Kull, Phys. Rep. 206, 197 (1991).
${ }^{26}$ D. H. Sharp, Physica D 12, 3 (1984).

${ }^{27}$ J. Glimm and D. H. Sharp, Phys. Rev. Lett. 64, 2137 (1990).

${ }^{28}$ D. Shvarts, U. Alon, D. Ofer, R. L. McCroy, and C. P. Verdon, Phys. Plasmas 2, 2465 (1995).

${ }^{29}$ K. I. Read, Physica D 12, 45 (1984).

${ }^{30}$ G. Dimonte and M. Schneider, Phys. Fluids 12, 304 (2000).

${ }^{31}$ D. L. Youngs, Physica D 12, 32 (1984).

${ }^{32}$ D. Oron, L. Arazi, D. Kartoon, A. Rikanati, U. Alon, and D. Shvarts, Phys. Plasmas 8, 2883 (2001).

${ }^{33}$ R. L. Panton, Incompressible Flow, 2nd ed. (Wiley, New York, 1996).

${ }^{34}$ X. L. Li, Phys. Fluids 8, 336 (1996). 